\title{
Microgrid Optimal Scheduling With Chance-Constrained Islanding Capability
}

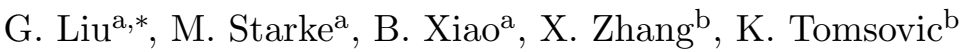 \\ ${ }^{a}$ Power and Energy Systems Group, Oak Ridge National Laboratory, One Bethel Valley \\ Road, P.O. Box 2008, MS-6070, Oak Ridge, TN 37831-6070, USA \\ ${ }^{b}$ Department of Electrical Engineering and Computer Science, The University of \\ Tennessee, Knoxville, 1520 Middle Drive, Knoxville, TN 37996, USA
}

\begin{abstract}
Abstract - To facilitate the integration of variable renewable generation and improve the resilience of electricity supply in a microgrid, this paper proposes an optimal scheduling strategy for microgrid operation considering constraints of islanding capability. A new concept, probability of successful islanding (PSI), indicating the probability that a microgrid maintains enough spinning reserve (both up and down) to meet local demand and accommodate local renewable generation after instantaneously islanding from the main grid, is developed. The PSI is formulated as mixed-integer linear program using multi-interval approximation taking into account the probability distributions of forecast errors of wind, PV and load. With the goal of minimizing the total operating cost while preserving user specified PSI, a chance-constrained optimization problem is formulated for the optimal scheduling of mirogrids and solved by mixed integer linear programming (MILP). Numerical simulations on a microgrid consisting of a wind turbine, a PV panel, a fuel cell, a micro-turbine, a diesel generator and a battery demonstrate the effectiveness of the proposed scheduling strategy. The relationship between PSI and various factors are verified.
\end{abstract}

Keywords: Microgrid, optimal scheduling, spinning reserve, islanding capability, chance constraints, mixed-integer linear programming (MILP)

\footnotetext{
${ }^{*}$ Corresponding author. Tel.: +1 865241 9732; fax.: +1 8655749329.

Email addresses: liug@ornl.gov (G. Liu), starkemr@ornl.gov (M. Starke), xiaob@ornl.gov (B. Xiao), xzhang46@utk.edu (X. Zhang), tomsovic@utk.edu (K. Tomsovic)
} 


\section{Nomenclature}

The main symbols used in this paper are defined below. Others will be defined as required in the text. A $\triangle$ indicates forecast error for the variable while ${ }^{\wedge}$ indicates the forecast value.

\subsection{Indices}

$i \quad$ Index of dispatchable generators, running from 1 to $N_{G}$.

$j \quad$ Index of demands, running from 1 to $N_{D}$.

$b \quad$ Index of battery storage devices, running from 1 to $N_{B}$.

$t \quad$ Index of time periods, running from 1 to $N_{T}$.

$m \quad$ Index of energy blocks offered by generators, running from 1 to $N_{I}$.

$l \quad$ Index of probability intervals, running from 1 to $N_{L}$.

0.2. Variables

0.2.1. Binary Variables

$u_{i t} \quad 1$ if unit $i$ is scheduled on during period $t$ and 0 otherwise.

$u_{b t}^{\mathrm{C}} \quad 1$ if battery $b$ is scheduled charging at period $t$ and 0 otherwise.

$u_{b t}^{\mathrm{D}} \quad 1$ if battery $b$ is scheduled discharging at period $t$ and 0 otherwise.

$b_{t l}^{\mathrm{U}}, b_{t l}^{\mathrm{D}} \quad$ Binary indicators of probability interval $l$ during period $t$.

\subsubsection{Continuous Variables}

$p_{i t}(m) \quad$ Power output scheduled from the $m$-th block of energy offer by dispatchable unit $i$ during period $t$. Limited to $p_{i t}^{\max }(m)$.

$P_{i t} \quad$ Power output scheduled from unit $i$ during period $t$.

$P_{t}^{\mathrm{PCC}} \quad$ Exchanged power at PCC during period $t$.

$P_{b t}^{\mathrm{C}}, P_{b t}^{\mathrm{D}} \quad$ Charging/discharging power of battery $b$ during period $t$.

$P_{b t} \quad$ Output power of battery $b$ during period $t$.

$S O C_{b t} \quad$ State of charge of battery $b$ during period $t$.

$R_{i t}^{\mathrm{U}}, R_{i t}^{\mathrm{D}} \quad$ Up- and down-spinning reserve of unit $i$ during period $t$.

$R_{b t}^{\mathrm{U}}, R_{b t}^{\mathrm{D}} \quad$ Up- and down-spinning reserve of battery $b$ during period $t$.

$\mathrm{PSI}_{t} \quad$ Probability of successful islanding during period $t$. 
0.3. Constants

$\lambda_{i t}(m) \quad$ Marginal cost of the $m$-th block of energy offer by dispatchable unit $i$ during period $t$.

$C_{b t} \quad$ Degradation cost of battery $b$ during period $t$.

$\lambda_{t}^{\mathrm{PCC}} \quad$ Purchasing/selling price of energy from/to distribution grid during period $t$.

$A_{i} \quad$ Operating cost of dispatchable unit $i$ at the point of $P_{i}^{\min }$.

$Q_{i t}^{\mathrm{U}}, Q_{i t}^{\mathrm{D}} \quad$ Cost of up- and down-spinning reserve of unit $i$ during period $t$.

$Q_{b t}^{\mathrm{U}}, Q_{b t}^{\mathrm{D}} \quad$ Cost of up- and down-spinning reserve of battery $b$ at period $t$.

$P_{i}^{\max }, P_{i}^{\min }$ Maximum/minimum output of DG $i$.

$P_{t}^{\mathrm{W}} \quad$ Wind turbine power output during period $t$.

$P_{t}^{\mathrm{PV}} \quad \mathrm{PV}$ power output during period $t$.

$P_{j t} \quad$ Power consumption scheduled for demand $j$ during period $t$.

$\triangle N_{t}^{D} \quad$ Net demand forecast error of microgrid during period $t$.

$\mu_{t}, \sigma_{t} \quad$ Mean and standard deviation of $\triangle N_{t}^{D}$.

PSI $^{\text {req }} \quad$ PSI requirements of microgrid operators.

$P_{b}^{\mathrm{C}, \max }, P_{b}^{\mathrm{D}, \max }$ Maximum charging/discharging power of battery $b$.

$S O C_{b t}^{\max }, S O C_{b t}^{\min }$ Maximum/minimum state of charge of battery $b$ during period $t$.

$\eta_{b}^{\mathrm{C}}, \eta_{b}^{D} \quad$ Battery charging/discharging efficiency factor.

$\pi_{l} \quad$ Probability of interval $l$.

$\triangle t \quad$ Time duration of each period.

$\tau \quad$ Amount of time available of DGs and batteries to ramp up/down their output to deliver the reserve. 


\section{Introduction}

A microgrid can be defined as a low voltage distribution network comprising various distributed energy resources (DERs), storage devices, and responsive loads that can be operated in both grid-connected and islanded modes [1]. It is connected to the main distribution network at the Point of Common Coupling (PCC), importing/exporting power from/to the distribution network as well as providing ancillary services, such as, voltage support, to the main distribution grid that a conventional end-user system cannot [2]-[4]. For customers, a microgrid can provide low-cost and environmental friendly energy. More importantly, a microgrid can significantly improve the reliability of local electricity supply by separating itself seamlessly from the main grid during an external disturbance, and operating in islanded mode with high efficiency and environmental friendly self-supply [5]. Due to such benefits, the microgrid has attracted growing attention from both academia and industry [6].

A master controller usually performs the scheduling of microgrids in both grid-connected and islanded modes. The master controller determines the optimal dispatch of DERs and exchanged power between the microgrid and the distribution utility through PCC that minimizes total operating cost subjected to various technical, reliability and operating constraints. Considerable efforts have been devoted to the optimal scheduling and management of microgrids [7]. Scheduling methods for a microgrid in islanded modes are proposed in [8]-[11]. Nevertheless, more research and published works are devoted to the scheduling of microgrids in grid-connected mode [12]-[17]. In particular, deterministic models are used in [12] and [13], while stochastic programming models have been developed in [14]-[16]. A hybrid stochastic/robust programming model for microgrid scheduling is proposed in [17]. The uncertainty management of the future power systems and the methods for managing the wind power intermittency has been reviewed in [18]-[19].

In the above literature, the scheduling models are mostly downsized version of the unit commitment (UC) problem in the bulk power systems since both problems are to determine the least cost dispatch of available resources to meet the forecasted demand while satisfying prevailing operational constraints [20]-[21]. However, the microgrid scheduling problem is considerably different from the UC problem due to the unique features of the microgrid. One of the key features of a microgrid is its ability to separate itself from the distribution utility (e.g., during disturbances or blackout periods experienced by utilities) in order to continue supplying loads in its own islanded portion. However, the transition of microgrid from grid-connected mode to islanded mode is usually associated with certain excessive load (or genera- 
tion). This is mainly because the exchanged power between the microgrid and distribution utility through PCC is forced to zero during the microgrid islanding transition. This may also occur for poor load scheduling, inaccurate forecasting of renewable generation, and so on. Under these conditions, the microgrid frequency drops or increases rapidly due to load and generation imbalance. To save the microgrid from a complete collapse and rebuild the balance between load and generation after islanding, maintaining an appropriate level of up- and down-spinning reserve is still necessary for microgrid while in grid-connected mode, although normal operation assumes the main grid acts as an infinite bus to provide frequency and voltage regulation. Therefore, the major difference between UC and microgrid scheduling is that microgrid islanding requirements should be considered in microgrid scheduling in case the main grid power is not available. In addition, renewable generation sources and energy storage systems have major roles in the microgrid operation due to their considerable size compared to local loads [22]. Furthermore, due to the limited capacity and the proximity of load and generation in a microgrid, the network is typically not the limiting constraint, and can be neglected. With these differences, developing new microgrid scheduling strategy capable of taking into consideration islanding capability of microgrids and probabilistic characteristics of load and renewable energy is of fundamental importance.

Accordingly, microgrid scheduling with islanding capability constraints has been proposed recently [22]-[24]. In [22], a microgrid optimal scheduling model considering multi-period islanding constraints was proposed. The model guarantees the generation adequacy of the microgrid in the islanded mode operation for certain specified duration in case of any unpredicted disconnection from the main grid. However, the uncertainty of renewable resources has been neglected. A new probability-based concept, probability of self-sufficiency (PSS), is proposed to indicate the probability that the microgrid is capable of meeting local demand in islanded mode in [23]. The probabilistic power balance constraint is reformulated into mixed-integer linear form and explicitly included in the microgrid scheduling model. However, this model only works under the assumption of normal distribution of net demand forecast error. In [24], a probabilistic methodology for estimating spinning reserve requirement in microgrids based on the tradeoff between reliability and economics is proposed. Nevertheless, the model is actually the downsized version of the reserve quantification problem in the bulk power systems. The special characteristics of microgrids, such as islanding capability, have been neglected. In addition, only up-spinning reserve is considered in [23] and [24]. In fact, over-generation in a microgrid after islanding is also 
a frequent and important issue considering various ownership of generating resources in microgrids.

In view of the shortcomings of the existing microgrid scheduling methods, a new optimal scheduling strategy for microgrid operation considering probabilistic constraints of successful islanding is proposed in this paper. Considering the uncertainty of renewable generation and power at the PCC, a new concept, probability of successful islanding (PSI), is proposed to indicate the probability that a microgrid is maintaining adequate up- and down-spinning reserve to meet local demand and accommodate local renewable generation after instantaneous islanding from the main grid. A new microgrid optimal scheduling strategy with chance-constrained islanding capability is developed. The proposed scheduling strategy allows for successful islanding of a microgrid with specified probability. The main contributions of this paper are as follows:

1. To quantify the probability of microgrid meeting local demands and accommodate renewable generation, the probability of successful islanding (PSI) is proposed and approximated in mixed-integer linear form.

2. As an innovative contribution, our proposed formulation of PSI considers both the up- and down-spinning reserve. In particular, it is compatible with any probability distribution of the uncertainties.

3. A new scheduling strategy with chance-constrained islanding capability is developed to ensure successful islanding of a microgrid with a specified probability.

The rest of this paper is organized as follows. In Section II, the microgrid scheduling strategy with deterministic islanding capability is presented. Based on this model, the new concept, PSI is formulated considering forecast errors of renewable resources and load as well as power at PCC in Section III. A mixed integer linear approximation of the nonlinear PSI expression is proposed and the microgrid scheduling strategy with probabilistic islanding capability is formulated. In Section IV, results of numerical simulations are presented. Finally, conclusions are given in Section V.

\section{Microgrid Scheduling With Deterministic Islanding Capability}

\subsection{Component Models}

The microgrid considered in this paper consists of distributed generators (e.g., diesel generators, microturbines and fuel cells), renewable generation (e.g.. wind turbines and PV panels), energy storage (e.g., battery systems) and local demands. The distributed generators are considered as 
dispatchable units, which can be controlled by a microgrid master controller to provide both power and reserve. Depending on unit type, dispatchable units are subject to various constraints, such as, capacity limits, minimum power output limits, ramping rates, minimum on/off time, and so on. In contrast, renewable generation, such as, wind turbines and PV panels, are taken as non-dispatchable units, which depend on the meteorological conditions of wind speed, temperature and solar irradiance. Thus, renewable generation is subject to variability. Although renewable generation integrated with advanced power electronic inverters could reduce their power output easily, they are not considered as reserve provider here due to uncertain availability. Extensive research has been done on wind and PV power forecasting [25]-[27]. In this section, both wind and PV power forecast error are modeled as independent normally distributed random variables [23]. Due to the intermittent and variable characteristics of renewable generation, they are commonly paired with energy storage systems, which can mitigate the intermittent renewable generation, shift loads and provide both up- and down-spinning reserve. The load forecast error is assumed to follow a normal distribution and be independent of renewable generation forecast [24], [28]. Due to the limited size of microgrid, relatively large standard deviations are used for both renewable generation and load forecast errors.

\subsection{Problem Formulation}

This subsection describes the model of a microgrid scheduling strategy with deterministic islanding capability. In the context of microgrids with dispatchable and undispatchable generation as well as electrical energy storage (e.g., batteries) integration, the objective aims at minimizing the total operation cost, including generation cost and spinning reserve cost of local resources as well as purchasing cost of energy from main grid. The decision variables include on/off status and power output of units, charging/discharging power of batteries, purchasing/selling power at PCC as well as the up- and down-spinning reserve provided by all units and batteries. The objective function is shown in (1). Specifically, the first and second line is the fuel cost of DGs (including DGs start-up cost $S U_{i t}$ ); the third line is the energy purchasing/selling cost/benefit from distribution grid; the fourth line is the battery degradation cost, the fifth and sixth lines are cost of up- and down-spinning reserve from both DGs and batteries. All terms are in mixed-integer linear form except the startup cost of generators (line 
2), which can be recast into mixed-integer linear form as in [29].

$$
\begin{aligned}
\min & \sum_{t=1}^{N_{T}} \sum_{i=1}^{N_{G}}\left[\sum_{m=1}^{N_{I}} \lambda_{i t}(m) p_{i t}(m)+A_{i} u_{i t}\right] \\
& +\sum_{t=1}^{N_{T}} \sum_{i=1}^{N_{G}} S U_{i t}\left(u_{i t}, u_{i, t-1}\right) \\
& +\sum_{t=1}^{N_{T}} \lambda_{t}^{\mathrm{PCC}} P_{t}^{\mathrm{PCC}} \\
& +\sum_{t=1}^{N_{T}} \sum_{b=1}^{N_{B}} C_{b t}\left(P_{b t}^{\mathrm{C}}+P_{b t}^{\mathrm{D}}\right) \\
& +\sum_{t=1}^{N_{T}} \sum_{i=1}^{N_{G}}\left(Q_{i t}^{\mathrm{U}} R_{i t}^{\mathrm{U}}+Q_{i t}^{\mathrm{D}} R_{i t}^{\mathrm{D}}\right) \\
& +\sum_{t=1}^{N_{T}} \sum_{b=1}^{N_{B}}\left(Q_{b t}^{\mathrm{U}} R_{b t}^{\mathrm{U}}+Q_{b t}^{\mathrm{D}} R_{b t}^{\mathrm{D}}\right)
\end{aligned}
$$

The objective function is subject to the following constraints:

$$
\begin{gathered}
P_{i t}=\sum_{m=1}^{N_{I}} p_{i t}(m)+u_{i t} P_{i}^{\min } \quad \forall i, \forall t \\
0 \leq p_{i t}(m) \leq p_{i t}^{\max }(m) \quad \forall i, \forall t, \forall m \\
P_{i}^{\min } u_{i t} \leq P_{i t} \leq P_{i}^{\max } u_{i t} \quad \forall i, \forall t \\
R_{i t}^{\mathrm{U}} \leq P_{i}^{\max } u_{i t}-P_{i t} \quad \forall i, \forall t \\
R_{i t}^{\mathrm{U}} \leq u_{i t} R_{i}^{\mathrm{U}, \max } \quad \forall i, \forall t \\
R_{i t}^{\mathrm{D}} \leq P_{i t}-P_{i}^{\min } u_{i t} \quad \forall i, \forall t \\
R_{i t}^{\mathrm{D}} \leq u_{i t} R_{i}^{\mathrm{D}, \max } \tau \quad \forall i, \forall t \\
0 \leq P_{b t}^{\mathrm{C}} \leq P_{b}^{\mathrm{C}, \max } u_{b t}^{\mathrm{C}} \quad \forall b, \forall t \\
0 \leq P_{b t}^{\mathrm{D}} \leq P_{b}^{\mathrm{D}, \max } u_{b t}^{\mathrm{D}} \quad \forall b, \forall t \\
u_{b t}^{\mathrm{C}}+u_{b t}^{\mathrm{D}} \leq 1 \quad \forall b, \forall t \\
S O C_{b, t-1}+P_{b t}^{\mathrm{C}} \eta_{b}^{\mathrm{C}} \triangle t-P_{b t}^{\mathrm{D}} \frac{1}{\eta_{b}^{\mathrm{D}}} \triangle t \quad \forall b, \forall t \\
S O C_{b t}^{\min } \leq S O C_{b t} \leq S O C_{b t}^{\max } \quad \forall b, \forall t \\
P_{b t}=P_{b t}^{\mathrm{D}}-P_{b t}^{\mathrm{C}} \quad \forall b, \forall t \\
R_{b t}^{\mathrm{U}} \leq P_{b}^{\mathrm{D}, \max }-P_{b t} \quad \forall b, \forall t
\end{gathered}
$$




$$
\begin{gathered}
R_{b t}^{\mathrm{U}} \leq \eta_{b}^{\mathrm{D}}\left(S O C_{b t}-S O C_{b t}^{\mathrm{min}}\right) / \tau \quad \forall b, \forall t \\
R_{b t}^{\mathrm{D}} \leq P_{b}^{\mathrm{C}, \mathrm{max}}+P_{b t} \quad \forall b, \forall t \\
R_{b t}^{\mathrm{D}} \leq 1 / \eta_{b}^{\mathrm{C}}\left(S O C_{b t}^{\max }-S O C_{b t}\right) / \tau \quad \forall b, \forall t \\
\sum_{i=1}^{N_{G}} P_{i t}+P_{t}^{N_{\mathrm{W}}}+P_{t}^{\hat{\mathrm{P}}}+P_{t}^{\mathrm{PCC}}+\sum_{b=1}^{N_{B}} P_{b t}^{\mathrm{D}}-\sum_{b=1}^{N_{B}} P_{b t}^{\mathrm{C}}=\sum_{j=1}^{N_{D}} \hat{P}_{j t} \quad \forall t \\
-\sum_{i=1}^{N_{G}} R_{i t}^{\mathrm{D}}-\sum_{b=1}^{N_{B}} R_{b t}^{\mathrm{D}} \leq P_{t}^{\mathrm{PCC}}+\triangle N_{t}^{D} \leq \sum_{i=1}^{N_{B}} R_{i t}^{\mathrm{U}}+\sum_{b=1}^{N_{D}} R_{b t}^{\mathrm{U}} \forall t \\
\triangle N_{t}^{D}=\sum_{j=1}^{\mathrm{P}} \triangle P_{j t}-\triangle P_{t}^{\mathrm{W}}-\triangle P_{t}^{\mathrm{PV}} \quad \forall t
\end{gathered}
$$

For DGs, constraints (2) and (3) approximate the production cost of dispatchable generators by blocks [30]. Constraint (4) forces the output of DG to be zero if it is not committed. The up-spinning reserve of DG is limited by the difference between its maximum capacity and current output in (5) and its ramping rate in (6). Similarly, the down-spinning reserve constraints are included in (7) and (8). For batteries, constraints (9) and (10) are the maximum charging/discharging power of a battery. These two states are mutually exclusive, which is ensured by (11). The battery state of charge (SOC) is defined by (12) and the limit of SOC is enforced by (13). The output power of a battery is represented in (14). Similar to DGs, the up-spinning reserve of a battery is constrained by the difference between its maximum discharging power and current output in (15) and the difference between its current SOC and minimum SOC in (16). In the same way, the down-spinning reserve constraints of a battery are included as in (17) and (18). The energy balance is enforced by (19). The total of the electricity produced by dispatchable generators, wind, PV, batteries and power purchased at PCC must be equal to the demand in the microgrid. The spinning reserve requirement is as (20), which guarantees adequate spinning reserve for successful islanding of the microgrid considering the forecast errors of demand, wind power and PV power. The net demand forecast error $\triangle N_{t}^{D}$ is formulated in (21). Additionally, each unit or demand is subject to its own operating constraints, such as, minimum up and down time, initial condition, and so on. See [20] for details about mathematical formulations of these constraints.

As mentioned in subsection 2.1, we assume both wind and PV power forecast error as well as demand forecast error can be modeled as independent normally distributed random variables. Thus, the net demand forecast error $\triangle N_{t}^{D}$ also follows normal distribution, i.e., $\triangle N_{t}^{D} \sim N\left(\mu_{t}, \sigma_{t}^{2}\right)$. Based 
on the three-sigma rule of thumb, the spinning reserve constraint (20) can replaced by (22) and (23) with a probability of $99.7 \%$ of successful islanding. In this paper, probability of successful islanding (PSI) is defined as the probability that a microgrid maintains enough spinning reserve (both up and down) to meet local demand and accommodate local renewable generation after instantaneously islanding from the main grid taking into account the probability distributions of forecast errors of wind, PV and load. In this case, $\mathrm{PSI}=99.7 \%$.

$$
\begin{gathered}
P_{t}^{\mathrm{PCC}}+\mu_{t}+3 \sigma_{t} \leq \sum_{i=1}^{N_{G}} R_{i t}^{\mathrm{U}}+\sum_{b=1}^{N_{B}} R_{b t}^{\mathrm{U}} t \\
-\sum_{i=1}^{N_{G}} R_{i t}^{\mathrm{D}}-\sum_{b=1}^{N_{B}} R_{b t}^{\mathrm{D}} \leq P_{t}^{\mathrm{PCC}}+\mu_{t}-3 \sigma_{t} t
\end{gathered}
$$

It should be pointed out that the deterministic model in this section can be extended to chance-constrained model as in [23] under the assumption that $\triangle N_{t}^{D}$ follows a symmetric probability distribution with analytical inverse distribution function. In the following section, a new formulation of PSI will be proposed. Particularly, it is compatible with any probability distribution or relative frequency distribution histogram of $\triangle N_{t}^{D}$.

In this paper, only main grid contingency events are taken into account since simultaneous failure of main grid and DERs have relatively small probabilities while consuming far more computational resources. Note the difference between a planning calculation where in general one needs to consider the possibility of multiple outages and units under maintenance. Nevertheless, the proposed algorithm can be expanded to taken into account of the reliability of DERs by adding scenarios of simultaneous failure of main grid and DERs [31].

It should also be noted that the network constraints of the microgrid have been neglected in the optimization model. There are two justifications for this simplification. First, a microgrid practical size may be limited to a few MVA [1]. IEEE draft standard P1547.4 specifies an upper limit of 10 MVA [32]. Due to the limited capacity and the proximity of load and generation in a microgrid, the network is typically not the limiting constraint. Second, the network model greatly complicate the day-ahead scheduling model without much likely benefit. Consideration of the network constraint in the shortterm or near real-time horizon, where most of the uncertainties have been realized, strikes us as more appropriate [33]. 


\section{Microgrid Scheduling With Chance-Constrained Islanding Ca- pability}

In Section 2, the net demand forecast error $\triangle N_{t}^{D}$ follows commonly used symmetrical probability distributions, such as normal distribution, while in practice, this is not true for microgrids with relatively concentrated footprint. Still, one can get the relative frequency distribution histogram of $\triangle N_{t}^{D}$ based on historical data. In this section, probability of successful islanding (PSI), is developed taking the probability distribution or relative frequency distribution histogram of $\triangle N_{t}^{D}$ into account and is reformulated into a mixed integer linear format using multi-interval approximation. A chance-constrained programming model for microgird scheduling is proposed and solved by mixed integer linear programming. The proposed model expands on the model in Section 2.

The PSI in time period $t$ can be expressed as (24). The microgrid is considered as successfully islanded if the net demand forecast error $\triangle N_{t}^{D} \in$ $\left[-\sum_{i=1}^{N_{G}} R_{i t}^{\mathrm{D}}-\sum_{b=1}^{N_{B}} R_{b t}^{\mathrm{D}}-P_{t}^{\mathrm{PCC}}, \sum_{i=1}^{N_{G}} R_{i t}^{\mathrm{U}}+\sum_{b=1}^{N_{B}} R_{b t}^{\mathrm{U}}-P_{t}^{\mathrm{PCC}}\right]$, where $\sum_{i=1}^{N_{G}} R_{i t}^{\mathrm{U}}+$ $\sum_{b=1}^{N_{B}} R_{b t}^{\mathrm{U}}-P_{t}^{\mathrm{PCC}}$ is the redundant up-spinning reserve after islanding and $-\sum_{i=1}^{N_{G}} R_{i t}^{\mathrm{D}}-\sum_{b=1}^{N_{B}} R_{b t}^{\mathrm{D}}-P_{t}^{\mathrm{PCC}}$ is the negative of the redundant downspinning reserve. PSI can be calculated by integrating the curve of $\triangle N_{t}^{D}$ over interval $\left[-\sum_{i=1}^{N_{G}} R_{i t}^{\mathrm{D}}-\sum_{b=1}^{N_{B}} R_{b t}^{\mathrm{D}}-P_{t}^{\mathrm{PCC}}, \sum_{i=1}^{N_{G}} R_{i t}^{\mathrm{U}}+\sum_{b=1}^{N_{B}} R_{b t}^{\mathrm{U}}-P_{t}^{\mathrm{PCC}}\right]$ for each time period $t$.

$\mathrm{PSI}_{t}=\mathrm{P}\left(-\sum_{i=1}^{N_{G}} R_{i t}^{\mathrm{D}}-\sum_{b=1}^{N_{B}} R_{b t}^{\mathrm{D}}-P_{t}^{\mathrm{PCC}} \leq \triangle N_{t}^{D} \leq \sum_{i=1}^{N_{G}} R_{i t}^{\mathrm{U}}+\sum_{b=1}^{N_{B}} R_{b t}^{\mathrm{U}}-P_{t}^{\mathrm{PCC}}\right)$

Note that equation (24) links the PSI with up- and down spinning reserve of a microgrid. With specified PSI requirement, a feasible region of spinning reserve, if existing, can be determined, whereas the microgrid PSI can be directly calculated with given spinning reserve and PCC power. Through this constraint, the spinning reserve requirements are automatically included into the microgrid scheduling model.

Apparently, direct calculation of PSI as in (24) is complex and nonlinear, and sometimes there is even no analytic formula. For this reason, a multiinterval approximation is proposed to estimate the PSI as shown in Fig. 1. On one hand, if $\triangle N_{t}^{D}$ follows a certain probability distribution, such as normal distribution (the black curve in Fig. 1), it can be discretized into $N_{L}$ odd number of intervals with $\pi_{l}$ as the probability of interval $l$. The width of each interval is $\sigma_{t}$. On the other hand, if the probability distribution of 
$\triangle N_{t}^{D}$ is unknown, the relative frequency distribution histogram of $\triangle N_{t}^{D}$ with $N_{L}$ odd number of intervals of equal length (such as the purple stairs in Fig. 1) can be developed based on historical data. In this scenario, $\sigma_{t}$ is the length of each interval determined by the users. For both cases, the mid-value of interval $l, \mu_{t}+\left(l-\left(N_{L}+1\right) / 2\right) \sigma_{t}$ is taken as the value of the whole interval.

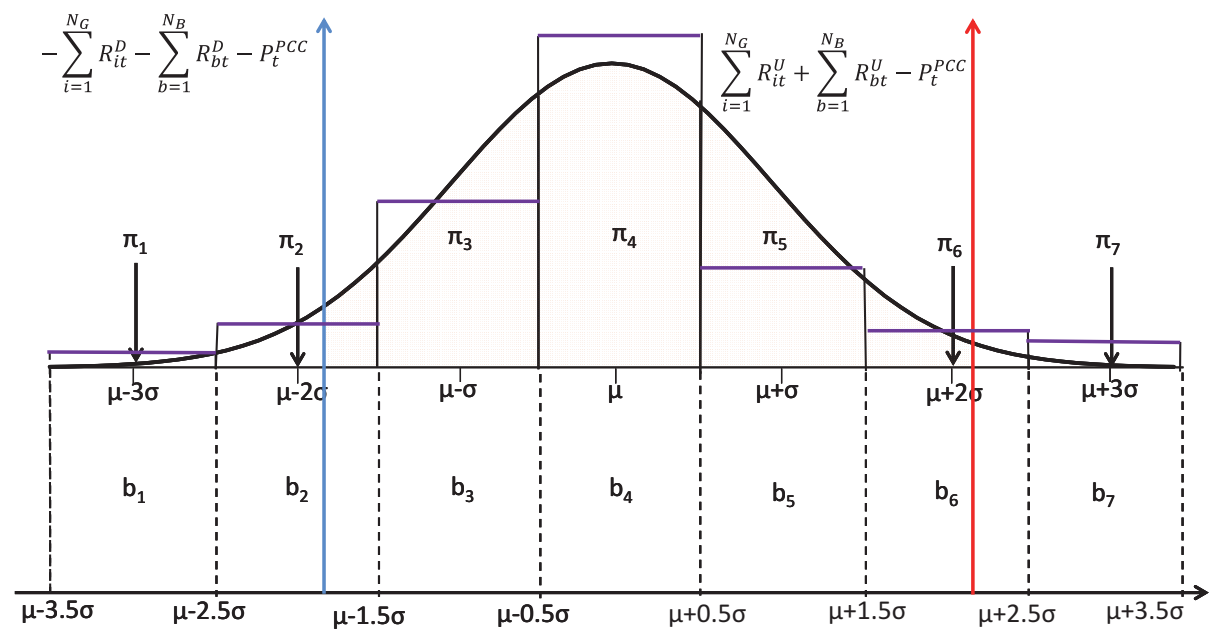

Figure 1: Seven-interval approximation of PSI

A seven-interval approximation of both cases is shown in Fig. 1. The red axis is the upper bound of $\triangle N_{t}^{D}$ that can be mitigated after islanding, and the blue axis is the lower bound of $\triangle N_{t}^{D}$ that can be mitigated after islanding. Both bounds are functions of $P_{t}^{\mathrm{PCC}}$ and spinning reserve. The integration of the area between the red axis and blue axis is the PSI. For each interval, if the mean value of this interval is located between the red axis and blue axis, the whole interval will be counted as capable of successful islanding. To distinguish intervals with islanding capability, two binary indicators $b_{t l}^{\mathrm{U}}$ and $b_{t l}^{\mathrm{D}}$ are introduced. The PSI is reformulated as:

$$
\mathrm{PSI}_{t}=\sum_{l=1}^{N_{L}}\left(b_{t l}^{\mathrm{U}}-b_{t l}^{\mathrm{D}}\right) \pi_{l} \quad \forall t
$$

The binary indicators $b_{t l}^{\mathrm{U}}$ and $b_{t l}^{\mathrm{D}}$ are associated with the realizations of net demand forecast error $\triangle N_{t}^{D}$, such that, 


$$
\begin{aligned}
& b_{t l}^{\mathrm{U}}=\left\{\begin{array}{ccc} 
& \text { if } \quad \sum_{i=1}^{N_{G}} R_{i t}^{\mathrm{U}} & +\sum_{b=1}^{N_{B}} R_{b t}^{\mathrm{U}}-P_{t}^{\mathrm{PCC}} \geq \mu_{t} \\
& & +\left(l-\frac{N_{L}+1}{2}\right) \sigma_{t}
\end{array}\right. \\
& b_{t l}^{\mathrm{D}}=\left\{\begin{array}{ccc} 
& \text { if } \quad-\sum_{i=1}^{N_{G}} R_{i t}^{\mathrm{D}}-\sum_{b=1}^{N_{B}} R_{b t}^{\mathrm{D}}-P_{t}^{\mathrm{PCC}} \geq \mu_{t} \\
& & +\left(l-\frac{N_{L}+1}{2}\right) \sigma_{t}
\end{array}\right.
\end{aligned}
$$

Expressions (26) and (27) ensure that $b_{t l}^{\mathrm{U}}-b_{t l}^{\mathrm{D}}$ equals to 1 if and only if the the realization of net demand forecast error associated with interval $l$ falls between the red axis and blue axis as shown in Fig. 1. The nonlinear expressions from (26) and (27) can be recast into an equivalent MILP form as given in (28)-(32).

$$
\begin{gathered}
{\left[\sum_{i=1}^{N_{G}} R_{i t}^{\mathrm{U}}+\sum_{b=1}^{N_{B}} R_{b t}^{\mathrm{U}}-P_{t}^{\mathrm{PCC}}-\mu_{t}-\left(l-\frac{N_{L}+1}{2}\right) \sigma_{t}\right] / M \leq b_{t l}^{\mathrm{U}}} \\
b_{t l}^{\mathrm{U}} \leq 1+\left[\sum_{i=1}^{N_{G}} R_{i t}^{\mathrm{U}}+\sum_{b=1}^{N_{B}} R_{b t}^{\mathrm{U}}-P_{t}^{\mathrm{PCC}}-\mu_{t}-\left(l-\frac{N_{L}+1}{2}\right) \sigma_{t}\right] / M \\
{\left[-\sum_{i=1}^{N_{G}} R_{i t}^{\mathrm{D}}-\sum_{b=1}^{N_{B}} R_{b t}^{\mathrm{D}}-P_{t}^{\mathrm{PCC}}-\mu_{t}-\left(l-\frac{N_{L}+1}{2}\right) \sigma_{t}\right] / M \leq b_{t l}^{\mathrm{D}}} \\
b_{t l}^{\mathrm{D}} \leq 1+\left[-\sum_{i=1}^{N_{G}} R_{i t}^{\mathrm{D}}-\sum_{b=1}^{N_{B}} R_{b t}^{\mathrm{D}}-P_{t}^{\mathrm{PCC}}-\mu_{t}-\left(l-\frac{N_{L}+1}{2}\right) \sigma_{t}\right] / M \\
M=\sum_{i=1}^{N_{G}} P_{i}^{\max }+\sum_{b=1}^{N_{B}} P_{b}^{\mathrm{C}, \text { max }}+P^{\mathrm{PCC}, \text { max }}+P^{\mathrm{W}, \text { max }}+P^{\mathrm{PV}, \max }
\end{gathered}
$$

Expressions (25) - (32) characterize a novel formulation of PSI considering the probability distributions of forecast errors of renewable generation and load. In particular, the PSI formulation and approximation proposed is compatible with any probability distribution of uncertainties (symmetric or asymmetric) [22]-[24]. To the best of our knowledge, no similar algorithm has been published in existing literature. Of course, one can divide the probability distribution of $\triangle N_{t}^{D}$ into smaller intervals, such as $\sigma_{t} / n$ by simply substituting $\sigma_{t} / n$ for $\sigma_{t}$ in (26) - (31). With small intervals, the result will be more accurate, but require more computational resources. Finally, the microgrid optimal scheduling with chance-constrained islanding capability can be formulated by substituting (20) - (23) with (25), (28) (32) and (33). The flowchart of the proposed chance-constrained microgrid 
scheduling model is shown in Fig. 2.

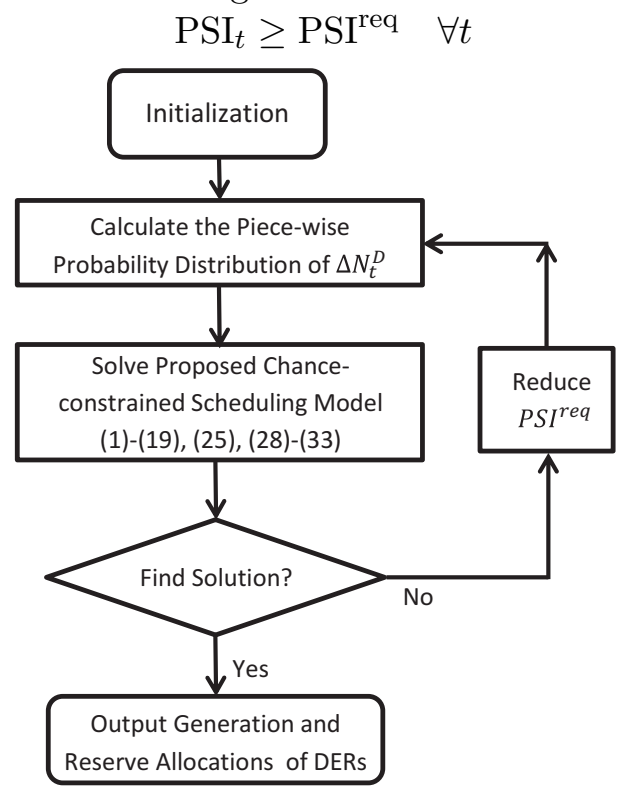

Figure 2: Flowchart of proposed change-constraint scheduling

It should be noted that the PSI proposed in this paper and the probability of self-sufficiency (PSS) in [23] in are different concepts. PSS indicates the probability that the microgrid is meeting local demand by its own generation resources in grid-connected mode. However, PSI is the probability of meeting local demand after instantaneous islanding through adjusting the output of local generation resources by deploying spinning reserve. In fact, PSS can be seen as a special case of PSI without considering the corrective control of microgrid after islanding. In extreme case with only battery and $\mathrm{PV}$, a microgrid during night time with $\mathrm{PSI}=100 \%$ may has $\mathrm{PSS}=0$ when the battery is idling, but ready to pick up all loads.

The introduction of chance-constrained islanding capability explicitly guarantees that the microgrid has adequate flexibility to meet local demand and accommodate local renewable generation after instantaneous islanding from the main grid with a certain probability specified by the microgrid operator. Thus, the improvement of supply reliability by the microgrid is clearly quantified. For example, the US Department of Energy (DOE), microgrid R\&D program specified the goal of reducing the outage time of required loads by more than $98 \%$ by 2020 . Correspondingly, PSI ${ }^{\text {req }}$ can be set as 0.98 in this case.

In a practical implementation, the calculated power output and reserve 
can be used to define the droop curves of inverters. For each inverter, the scheduled power plus up-spinning reserve corresponds to the lower bound of the frequency limit, while the scheduled power minus down-spinning reserve corresponds to the upper bound of the frequency limit. In grid-connected mode, the power output of inverter should be very close to the scheduled value since the grid frequency under normal condition is pretty stable. When suddenly islanded, the inverters adjust their output according to the predefined droop curve immediately. Thus, the exchanged power at PCC is mitigated and the balance between load and generation is regained after islanding with specified probability.

It should be noted that the reactive power and voltage limits have been neglected in the proposed model. Unlike real power, reactive power is usually compensated locally by capacitors and/or static VAR compensators (SVC). Efficient operation of the distribution network requires reducing the reactive power flow on feeders and PCC as much as possible. Under this circumstance, the reactive power and voltage are typically not the limiting constraints during microgrid islanding process.

\section{Case Studies}

\subsection{Test System Data}

The proposed microgrid scheduling strategy with chance-constrained islanding capability is demonstrated on the modified Oak Ridge National Laboratory (ORNL) Distributed Energy Control and Communication (DECC) lab microgrid test system as shown in Fig. 3. The modified system includes various DERs, including a wind turbine, PV panel, fuel cell, microturbine, diesel generator and battery. The parameters for the dispatchable generators are taken from [17]. For simplicity, the quadratic cost curves are converted into three-piece piece-wise linear cost curves. In addition, we assume that all units offer capacity cost of up and down spinning reserve at the rates of $30 \%$ of their highest incremental cost of producing energy [37]. Due to the relative small capacity of generators, their minimum up and down time is neglected.

The $60 \mathrm{~kW}$ wind turbine model is from [34]. Based on the wind speed forecast result, the corresponding power outputs of wind turbine are calculated as in Table 1. The generation cost of a wind turbine is assumed to be zero. The $60 \mathrm{~kW} \mathrm{PV} \mathrm{model} \mathrm{is} \mathrm{from} \mathrm{[35].} \mathrm{The} \mathrm{solar} \mathrm{irradiance} \mathrm{and} \mathrm{tempera-}$ ture are the measured data from [36]. The corresponding power outputs of PV are shown in Table 1. The forecast errors of wind power and PV power are assumed to be independent Gaussian distribution with zero mean with $15 \%$ standard deviation. The capacity of the battery is $100 \mathrm{kWh}$ with a 


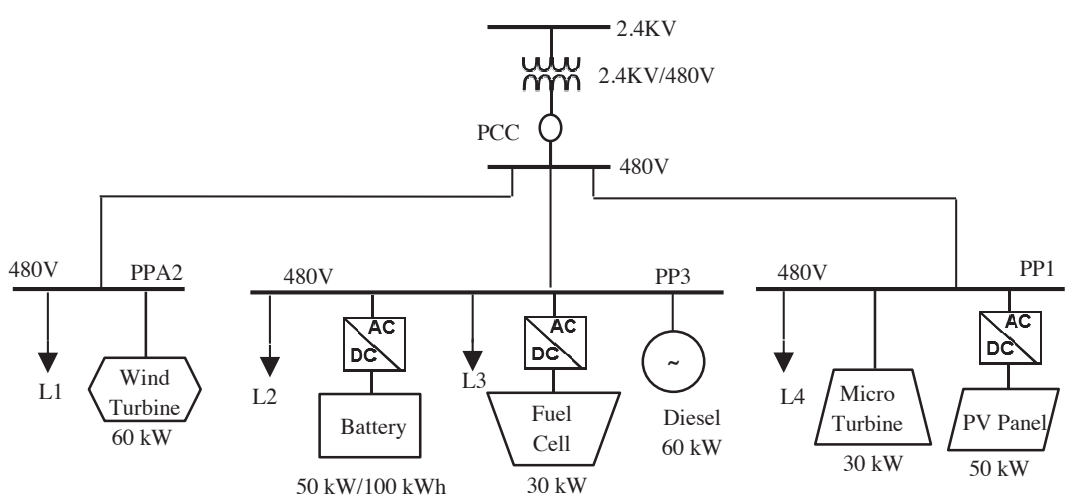

Figure 3: Modified ORNL DECC microgrid test system

Table 1: Forecast wind and PV power

\begin{tabular}{|c|c|c|c|c|c|}
\hline Hour & Wind $(\mathrm{kW})$ & PV $(\mathrm{kW})$ & Hour & Wind $(\mathrm{kW})$ & PV $(\mathrm{kW})$ \\
\hline 1 & 51.4829 & 0 & 13 & 23.5169 & 35.2199 \\
\hline 2 & 38.3711 & 0 & 14 & 39.4794 & 35.4594 \\
\hline 3 & 43.5590 & 0 & 15 & 35.7380 & 34.8303 \\
\hline 4 & 40.7514 & 0 & 16 & 18.0583 & 23.6244 \\
\hline 5 & 27.7421 & 0 & 17 & 24.2732 & 14.1823 \\
\hline 6 & 30.1540 & 0 & 18 & 26.2555 & 4.6705 \\
\hline 7 & 28.6452 & 0.1617 & 19 & 26.7732 & 0.1836 \\
\hline 8 & 23.3767 & 1.7726 & 20 & 26.2159 & 0 \\
\hline 9 & 21.7503 & 5.2978 & 21 & 32.8428 & 0 \\
\hline 10 & 34.8202 & 11.6044 & 22 & 36.0156 & 0 \\
\hline 11 & 27.1748 & 36.6382 & 23 & 37.2312 & 0 \\
\hline 12 & 30.1965 & 42.6778 & 24 & 44.1215 & 0 \\
\hline
\end{tabular}

maximum charging/discharging power of $50 \mathrm{~kW}$. The battery efficiency is assumed to be 0.9 . The minimum and maximum SOC of the battery is $25 \%$ and $95 \%$, respectively. The battery degradation cost is set as $0.02 \$ / \mathrm{kWh}$ and the up- and down reserve cost is assumed to be $40 \%$ of its degradation cost [16].

The analysis is conducted for a 24-hour scheduling horizon and each time interval is set to be one hour. The probability interval size is set as $\sigma_{t} / 5$. The forecast total demand and day-ahead market prices are shown in Table 2. The demand forecast error is assumed to be independent Gaussian distribution with zero mean and $3 \%$ of standard deviation.

All numerical simulations are coded in MATLAB and solved using the MILP solver CPLEX 12.6. The state-of-the-art branch-and-cut algorithm 
Table 2: Forecast load and day-ahead market prices

\begin{tabular}{|c|c|c|c|c|c|}
\hline Hour & Load $(\mathrm{kW})$ & $\begin{array}{c}\text { Price } \\
(\mathrm{ct} / \mathrm{kWh})\end{array}$ & Hour & Load $(\mathrm{kW})$ & $\begin{array}{c}\text { Price } \\
(\mathrm{ct} / \mathrm{kWh})\end{array}$ \\
\hline 1 & 124.7103 & 8.65 & 13 & 189.9998 & 26.82 \\
\hline 2 & 123.9769 & 8.11 & 14 & 187.0655 & 27.35 \\
\hline 3 & 126.9111 & 8.25 & 15 & 192.2006 & 13.81 \\
\hline 4 & 124.7103 & 8.10 & 16 & 194.4014 & 17.31 \\
\hline 5 & 128.3783 & 8.14 & 17 & 187.0655 & 16.42 \\
\hline 6 & 135.4320 & 8.13 & 18 & 185.5983 & 9.83 \\
\hline 7 & 146.7180 & 8.34 & 19 & 183.3975 & 8.63 \\
\hline 8 & 178.2624 & 9.35 & 20 & 187.0655 & 8.87 \\
\hline 9 & 186.3319 & 12.0 & 21 & 190.7334 & 8.35 \\
\hline 10 & 190.7334 & 9.19 & 22 & 181.9303 & 16.44 \\
\hline 11 & 195.8685 & 12.3 & 23 & 161.3898 & 16.19 \\
\hline 12 & 189.9998 & 20.7 & 24 & 134.9806 & 8.87 \\
\hline
\end{tabular}

combined with certain pre-processing and probing techniques are used to solve the MILP problems in CPLEX [38]. With a pre-specified duality gap of $0.1 \%$, the running time of the deterministic model is about 30 seconds on a $2.66 \mathrm{GHz}$ Windows-based PC with $4 \mathrm{G}$ bytes of RAM, while that of the proposed model is about 10 seconds on the same computer.

\subsection{Comparing Results of Deterministic and Chance-constrained Model}

It should be noted that the microgrid scheduling model with deterministic islanding capability is equivalent to the proposed chance-constrained model with $\mathrm{PSI}^{\mathrm{req}}=0.997$. The scheduling results of these two models are compared. The output of generators is compared in Fig. 4. The total upand down-spinning reserve are compared in Fig. 5 and the power at the PCC are compared in Fig. 6. As can be seen, the scheduling results of chance-constrained model with $\mathrm{PSI}^{\mathrm{req}}=0.997$ are almost the same as that of the deterministic model. Thus, the soundness of the proposed chanceconstrained model is validated.

It can be seen that the total up-spinning reserve and down-spinning reserve are generally complementary in Fig 5 . When high up-spinning reserve is required, the down-spinning reserve is low and vice versa. This can be explained by Fig. 1, where the sum of total up- and down-spinning reserve is the distance between the red and blue axis. In this case with $\mathrm{PSI}^{\mathrm{req}}=0.997$, it equals to $6 \sigma_{t}$. Thus, they are generally complementary to each other. In addition, the amounts of reserve are closely related to the power at the PCC. As can be seen in Fig. 5 and Fig. 6, when the microgrid is import- 
ing a large amount of power from PCC (e.g., hour 20-22), high up-spinning reserve is required in case of the loss of the power at the PCC. In contrast, when microgrid is exporting large amount of power through the PCC (e.g., hour 12-14), high down-spinning reserve is required in guard against over generation when losing the connection at the PCC.

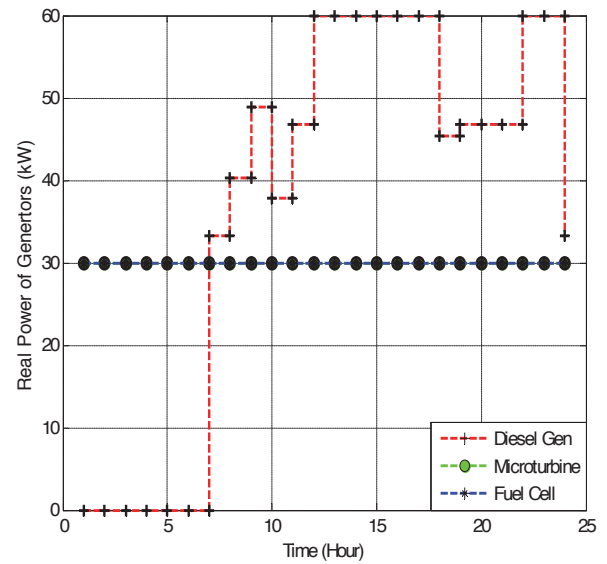

(a) Deterministic model

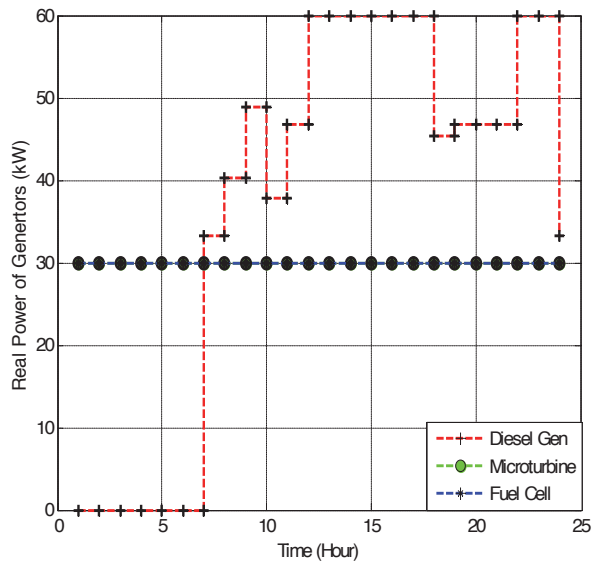

(b) Chance-constrained model

Figure 4: Comparison of generator outputs by deterministic and change-constrained model

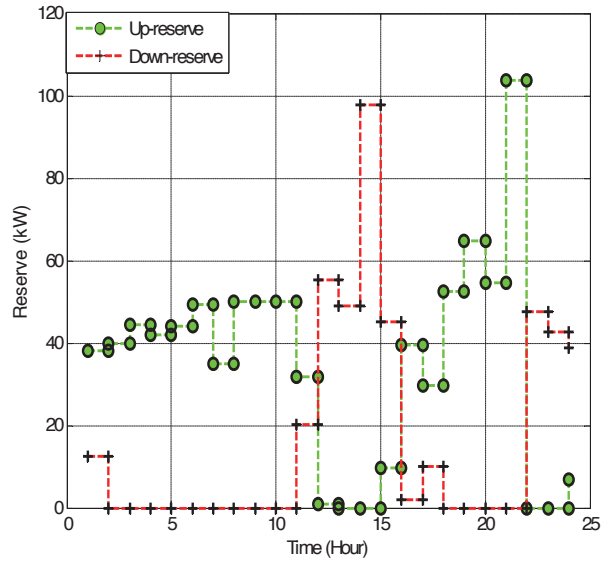

(a) Deterministic model

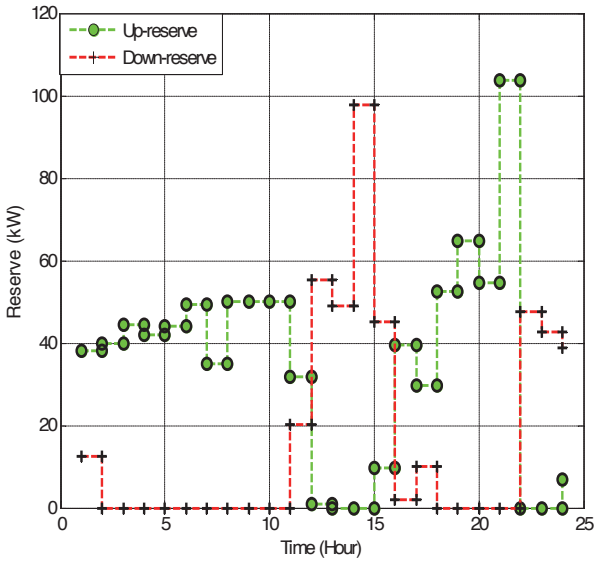

(b) Chance-constrained model

Figure 5: Comparison of total up- and down-spinning reserve by deterministic and change-constrained model 


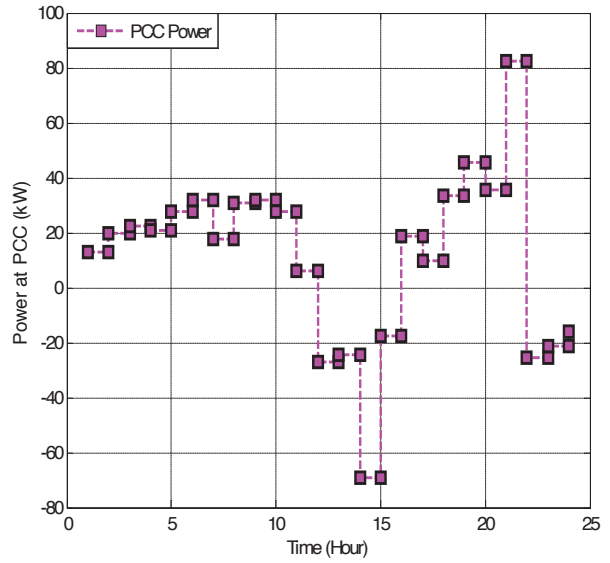

(a) Deterministic model

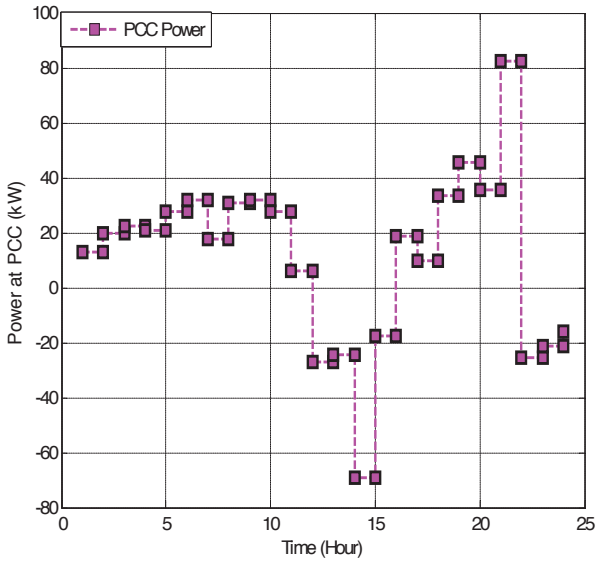

(b) Chance-constrained model

Figure 6: Comparison of power at PCC by deterministic and change-constrained model

\subsection{Sensitivity Analysis with Different Levels of PSI}

The results of chance-constrained microgrid scheduling with different PSI settings are compared in this subsection. To test the solution of the proposed chance-constrained microgrid scheduling with different PSI settings, a Monte-Carlo simulation method is used to generate 5,000 scenarios of renewable generation and loads. Both wind and PV power forecast errors as well as load forecast error are modeled as independent Gaussian distributed random variables with zero mean. For each scenario, power at PCC considering the realized forecast errors of renewable generation and loads are compared with the calculated up- and down-spinning reserve under certain PSI settings. If (20) is satisfied, this scenario is count as capable of successful islanding. The probability of successful islanding at different time intervals under various PSI settings are presented in Fig. 7. As observed, the calculated PSI are generally close to the corresponding PSI settings. Particularly, the multi-interval approximation is more accurate when the PSI setting is high. For example, when PSI setting is 0.9, the largest error between calculated PSI and PSI setting is less than 1.5\%. However, this error will be reduce to less than $0.15 \%$ when the PSI setting is 0.997 .

The total operating cost of the microgrid with different levels of PSI settings is compared in Fig. 8a. With increasing PSI, the total operating cost of the microgrid monotonically increases but in a highly nonlinear fashion. When the PSI increases from 0.4 to 0.9 , the total operating cost only increases $1.98 \%$. However, as PSI increases from 0.9 to 0.997 , the total operating cost increases $2.45 \%$. It indicates that a small increase of reliability 


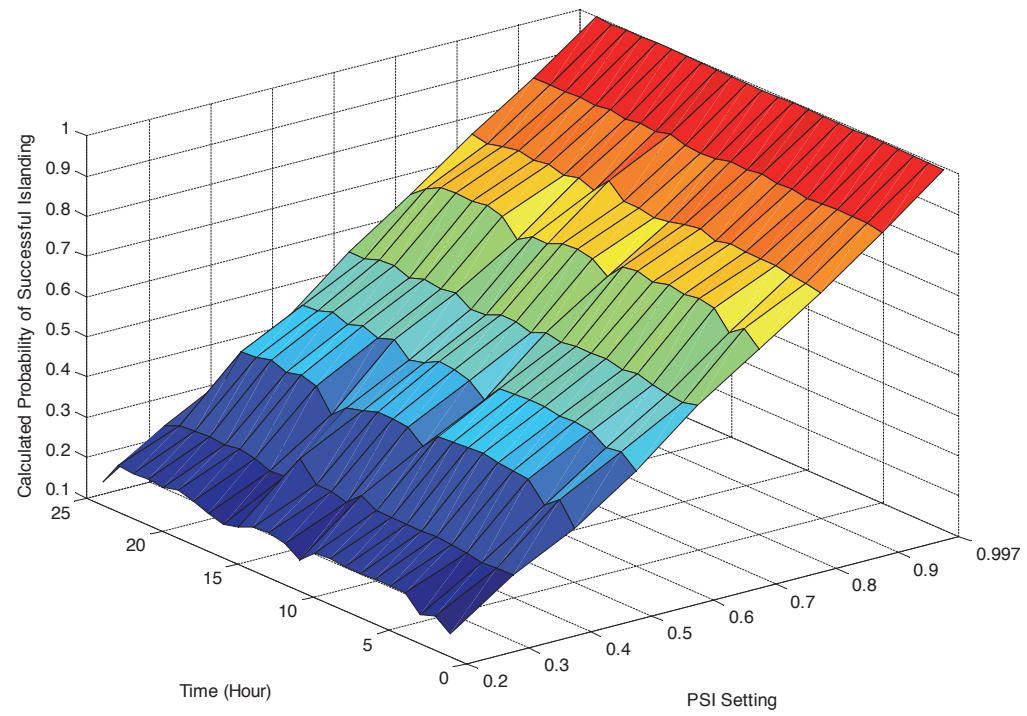

Figure 7: Calculated PSI with different levels of PSI settings

will require a significant increase of cost when the reliability of system is already high.

The power exchanged at PCC with different levels of PSI settings is compared in Fig. 8b. Generally, the exchanged (both imported and exported) power at PCC is decreasing with increasing PSI. This effect is much more obvious when the exchange power is high, e.g., hour 14, 18-23. This is because the imported/exported power at PCC has to be balanced by up-/downspinning reserve whenever the microgrid experiences an islanding condition. When the up-/down-spinning reserve reach their limits (e.g., hour 14 and 21, intervals with high exchanged power at PCC), the exchanged power at PCC will be reduced with increasing PSI.

With different levels of PSI requirements, the total up- and down-spinning reserve of the microgrid are compared in Fig. 9. Both up- and down-spinning reserve fluctuate significantly due to the forecast errors of renewable and load as well as the exchanged power at PCC. Therefore, unlike the bulk power system, it is not meaningful to keep a uniform reserve level for a microgrid. In addition, the total up-spinning reserve and down-spinning reserve are generally complementary. This has been explained earlier. Finally, note that the required both up- and down- spinning reserve increase with higher PSI. This increase is more obvious for intervals with high exchanged power at the PCC, e.g., hour 14 and 21. 


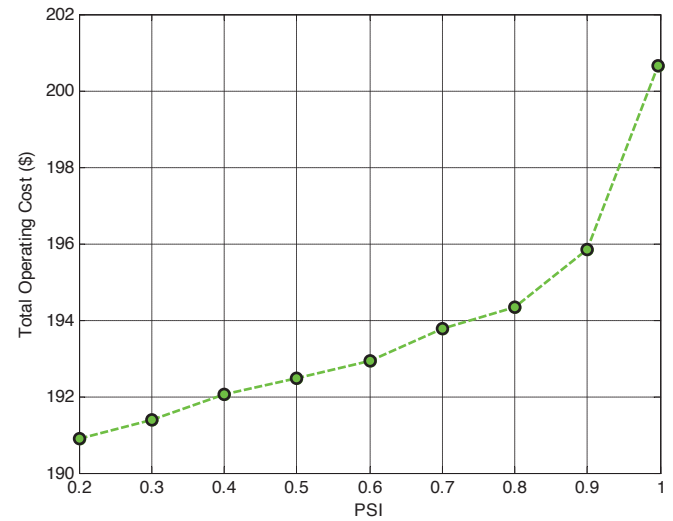

(a) Total operating cost

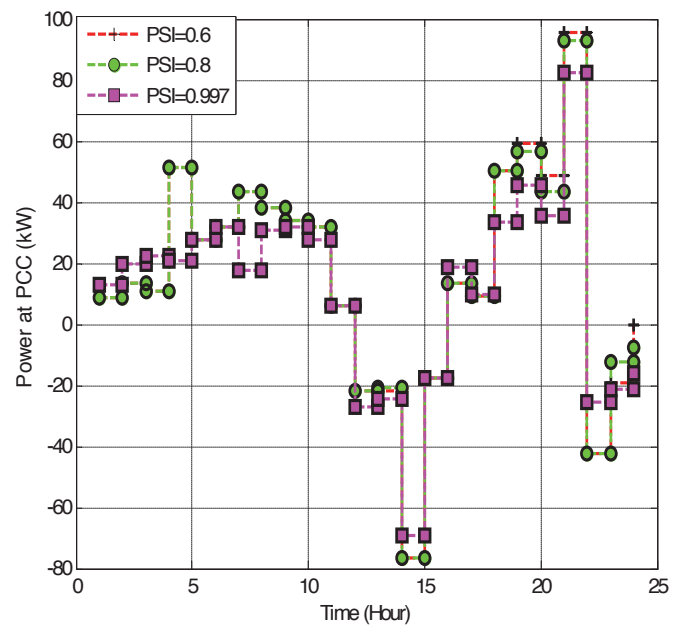

(b) Power at PCC

Figure 8: Comparison of total operating cost and exchanged power at PCC with different PSI settings

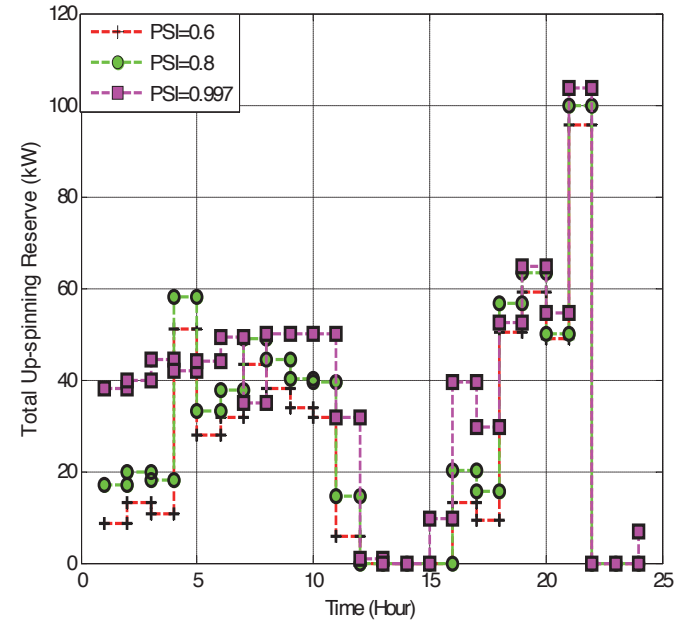

(a) Up-spinning Reserve

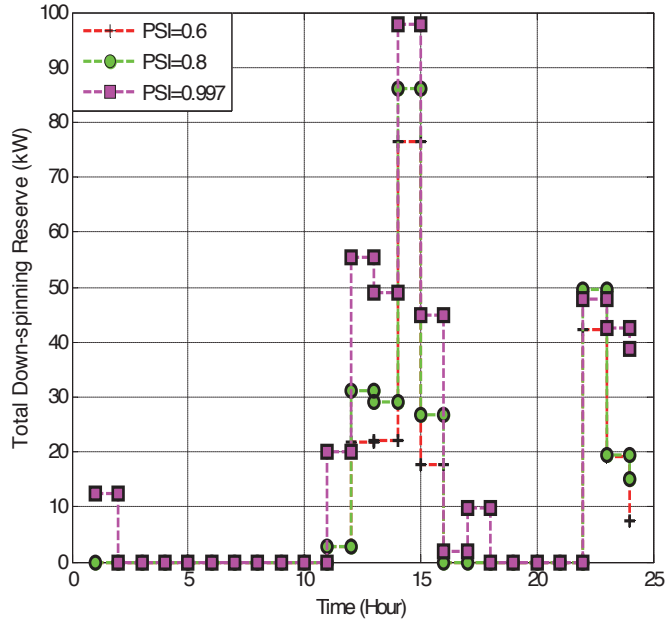

(b) Down-spinning Reserve

Figure 9: Comparison of total up- and down-spinning reserve with different levels of PSI 


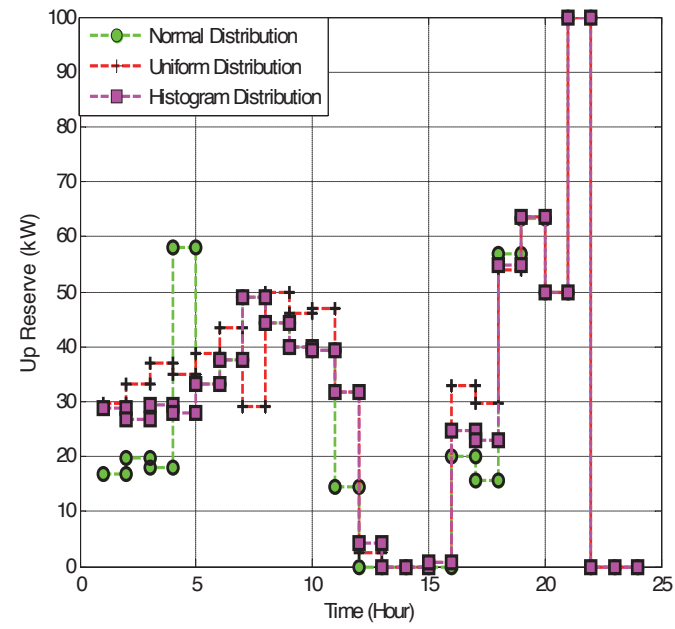

(a) Up-spinning Reserve

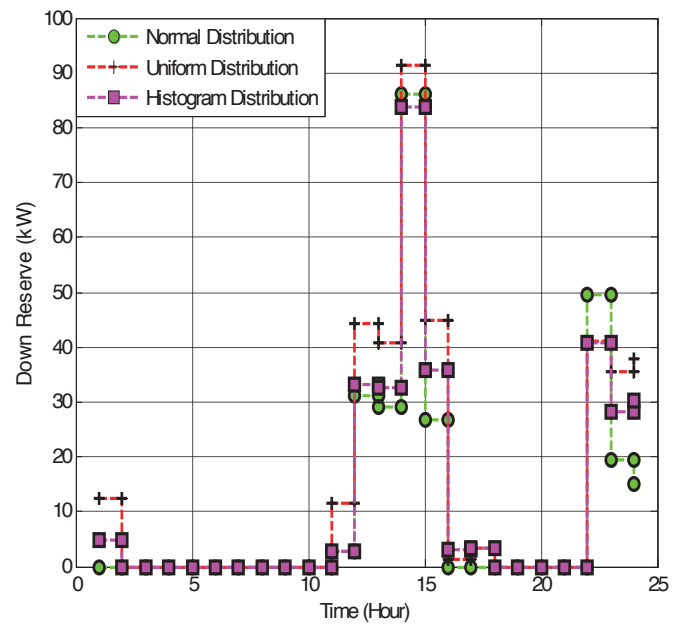

(b) Down-spinning Reserve

Figure 10: Comparison of total up- and down-spinning reserve with different probability distributions of net demand forecast error

\subsection{Comparing Results with Different Probability Distributions of Net De- mand Forecast Error}

The results of chance-constrained microgrid scheduling with different probability distributions of net demand forecast error $\triangle N_{t}^{D}$ are compared in this subsection. Three different distributions of $\triangle N_{t}^{D}$, including normal distribution, uniform distribution and a relative frequency distribution histogram as shown in Fig. 1 are analyzed. The PSI ${ }^{\text {req }}$ is set as 0.8 for all cases. For the relative frequency distribution histogram, the probability of the seven intervals is set as $[0.056,0.111,0.142,0.382,0.120,0.099,0.09]$. The calculated up-spinning reserve and down-spinning reserve of the microgrid are compared in Fig. 10. As can be seen, the probability distribution of net demand forecast error $\triangle N_{t}^{D}$ has significant effects on both up- and downspinning reserve requirements of the microgrid. The deterministic model in Section 2 requires that $\triangle N_{t}^{D}$ follows a symmetric probability distribution with analytical inverse distribution function, while robust optimization based approaches normally model uncertainties as independent, symmetric and bounded random variables with unknown distribution. By contrast, our proposed algorithm is compatible with any probability distribution of uncertainties (symmetric or asymmetric), thus more general and accurate. 


\section{Conclusions}

In this paper, a new microgrid scheduling strategy with chance-constrained islanding capability is developed to ensure successful islanding of a microgrid with a specified probability. To quantify the islanding capability of a microgrid, a new concept, probability of successful islanding (PSI) is proposed considering the uncertainty of renewable energy resources and load as well as exchanged power at PCC. Compared to the deterministic model, the proposed chance-constrained model has two advantages. First, it can explicitly guarantee the microgrid has sufficient flexibility to meet local demand and accommodate local renewable generation after instantaneously islanding from the main grid with certain probability specified by the microgrid operator. Second, it is more accurate and practical by considering probability distributions of forecast errors of wind, PV and load. Numerical simulations on a microgrid composed of a wind turbine, PV panel, fuel cell, micro-turbine, diesel generator and battery validated the effectiveness of proposed method. In addition, sensitivity analysis with different levels of PSI is included.

\section{Acknowledgements}

This manuscript has been authored by UT-Battelle, LLC under Contract No. DE-AC05-00OR22725 with the U.S. Department of Energy. The United States Government retains and the publisher, by accepting the article for publication, acknowledges that the United States Government retains a nonexclusive, paid-up, irrevocable, world-wide license to publish or reproduce the published form of this manuscript, or allow others to do so, for United States Government purposes. The Department of Energy will provide public access to these results of federally sponsored research in accordance with the DOE Public Access Plan(http://energy.gov/downloads/doe-publicaccess-plan).

This work also made use of Engineering Research Center Shared Facilities supported by the Engineering Research Center Program of the National Science Foundation and the Department of Energy under NSF Award Number EEC-1041877 and the CURENT Industry Partnership Program.

\section{References}

[1] The CERTS Microgrid Concept, CERTS Program Office, Oct. 2003. [Online]. Available: http://certs.lbl.gov/certs-der-micro.html.

[2] A. G. Madureira, J. A. Pecas Lopes, Coordinated voltage support in distribution networks with distributed generation and microgrids, IET Renew. Power Gen. 3(4) (2009) 439-454. 
[3] S. Beer, T. Gomez, D. Dallinger, I. Momber, C. Marnay, M. Stadler, J. Lai, An economic analysis of used electric vehicle batteries integrated into commercial building microgrids, IEEE Trans. Smart Grid 3(1) (2012) 517-525.

[4] A. G. Tsikalakis, N. D. Hatziargyriou, Centralized control for optimizing microgrids operation, IEEE Trans. Energy Convers. 23(1) (2008) 241-248.

[5] F. Katiraei, M. R. Iravani, P. W. Lehn, Micro-grid autonomous operation during and subsequent to islanding process, IEEE Trans. Power Del. 20(1) (2005) 248-257.

[6] M. Agrawal, A. Mittal, Microgrid technological activities across the globe: A review, Int. J. Res. Rev. Appl. Sci. 7(2) (2011) 147-152.

[7] W. Gu, Z. Wu, R. Bo, W. Liu, G. Zhou, W. Chen, A. Wu, Modeling, planning and optimal energy management of combined cooling, heating and power microgrid: A review, Int. J. Electr. Power Energy Syst. 54 (2014) 26-37.

[8] B. Belvedere, M. Bianchi, A. Borghetti, C. A. Nucci, M. Paolone, A. Peretto, A microcontroller-based power management system for standalone microgrids with hybrid power supply, IEEE Trans. Sustain. Energy 3(3) (2012) 422-431.

[9] H. Morais, P. Kadar, P. Faria, Z. A. Vale, H. M. Khodr, Optimal scheduling of a renewable micro-grid in an isolated load area using mixed-integer linear programming, Renew. Energy 35(1) (2010) 151-156.

[10] H. Babazadeh, W. Gao, Z. Wu, Y. Li, Optimal energy management of wind power generation system in islanded microgrid system, in: North Amer. Power Symp. (NAPS), September 2013, pp. 1-5.

[11] R. Palma-Behnke, C. Benavides, F. Lanas, B. Severino, L. Reyes, J. Llanos, D. Sez, A microgrid energy management system based on the rolling horizon strategy, IEEE Trans. Smart Grid 4(2) (2013) 996-1006.

[12] A. Sobu, G. Wu, Dynamic optimal schedule management method for microgrid system considering forecast errors of renewable power generations, in: IEEE Int. Conf. Power Syst. Tech. (POWERCON), November 2012, pp. 1-6.

[13] F. A. Mohamed, H. N. Koivo, System modelling and online optimal management of MicroGrid using Mesh Adaptive Direct Search, Int. J. Electr. Power Energy Syst. 32(5) (2010) 398-407.

[14] G. Cardoso, M. Stadler, A. Siddiqui, C. Marnay, N. DeForest, A. Barbosa-Pvoa, P. Ferro, Microgrid reliability modeling and battery scheduling using stochastic linear programming, Electr. Power Syst. Res. 103 (2013) 61-69.

[15] W. Su, J. Wang, J. Roh, Stochastic Energy Scheduling in Microgrids With Intermittent Renewable Energy Resources, IEEE Trans. Smart Grid 5(4) (2014) 1876-1883.

[16] D. T. Nguyen, L. B. Le, Optimal Bidding Strategy for Microgrids Considering Renewable Energy and Building Thermal Dynamics, IEEE Trans. Smart Grid 5(4) (2014) 1608-1620. 
[17] G. Liu, Y. Xu, K. Tomsovic, Bidding Strategy for Microgrid in Day-Ahead Market Based on Hybrid Stochastic/Robust Optimization, IEEE Trans. Smart Grid 7(1) (2016) 227-237.

[18] A. Alqurashi, A. H. Etemadi, A. Khodaei, Treatment of uncertainty for next generation power systems: State-of-the-art in stochastic optimization, Electr. Power Syst. Res. 141 (2016) 233-245.

[19] E. Rahimi, A. Rabiee, J. Aghaei, K. M. Muttaqi, A. E. Nezhad, On the management of wind power intermittency, Renew. Sustain. Energy Rev. 28 (2013) 643-653.

[20] M. Carrin, J. M. Arroyo, A computationally efficient mixed-integer linear formulation for the thermal unit commitment problem, IEEE Trans. Power Syst. 21(3) (2006) 1371-1378.

[21] M. Amini, A. Kargarian, O. Karabasoglu, ARIMA-based decoupled time series forecasting of electric vehicle charging demand for stochastic power system operation, Electr. Power Syst. Res. 140 (2016) 378-390.

[22] A. Khodaei, Microgrid Optimal Scheduling With Multi-Period Islanding Constraints,IEEE Trans. Power Syst. 29(3) (2014) 1383-1392.

[23] B. Zhao, Y. Shi, X. Dong, W. Luan, J. Bornemann, Short-Term Operation Scheduling in Renewable-Powered Microgrids: A Duality-Based Approach, IEEE Trans. Sustain. Energy 5(1) (2014) 209-217.

[24] M. Q. Wang, H. B. Gooi, Spinning Reserve Estimation in Microgrids, IEEE Trans. Power Syst. 26(3) (2011) 1164-1174.

[25] J. Carta, P. Ramrez, S. Velzquez, A review of wind speed probability distributions used in wind energy analysis, Renew. Sustain. Energy Rev. 13(5) (2009) 933-955.

[26] S. S. Soman, H. Zareipour, O. Malik, P. Mandal, A review of wind power and wind speed forecastin $\mathrm{g}$ methods with different time horizons, in: North Amer. Power Symp. (NAPS), Arlington, TX, USA, 2010, pp. 1-8.

[27] P. Bacher, H. Madsen, H. A. Nielsen, Online short-term solar power forecasting, Solar Energy 83 (2009) 1772-1783.

[28] K. Boroojeni, M. Amini, S. Bahrami, S. Iyengar, A. Sarwat, O. Karabasoglu, A novel multi-time-scale modeling for electric power demand forecasting: From short-term to medium-term horizon, Electr. Power Syst. Res. 142 (2017) 58-73.

[29] M. Ortega-Vazquez, Optimizing the spinning reserve requirements, Sch. Elect. Electron. Eng. Manchester, U.K.: Univ. Manchester, 2006, pp. 1-219 [Online]. Available: http://www.eee.manchester.ac.uk/research/groups/eeps/publications/reportstheses/aoe/ortegavazquez_PhD_2006.pdf.

[30] F. Aminifar, M. Fotuhi-Firuzabad, M. Shahidehpour, Unit commitment with probabilistic spinning reserve and interruptible load considerations, IEEE Trans. Power Syst. 24 (1) (2009) 388-397. 
[31] G. Liu, K. Tomsovic, Quantifying Spinning Reserve in Systems With Significant Wind Power Penetration, IEEE Trans. Power Syst. 27 (4) (2012) 2385-2393.

[32] IEEE Draft Guide for Design, Operation, and Integration of Distributed Resource Island Systems with Electric Power Systems, IEEE P1547.4/D12, April 27, 2011, pp. 1-50.

[33] G. Liu, M. Starke, X. Zhang, K. Tomsovic, A MILP-Based Distribution Optimal Power Flow Model for Microgrid Operation, in: IEEE PES Gen. Meet., Boston, MA, USA, July 2016, pp. 1-5.

[34] [Online]. Available: http://en.wind-turbine-models.com/turbine/262/tacke/tw-60.

[35] MSX-60 and MSX-64 Photovoltaic Modules. [Online]. Available: https://www.smud.org/en/about-smud/environment/renewableenergy/documents/solar-regatta-photovoltaic-specs.pdf.

[36] [Online]. Available: http://www.nrel.gov/midc/ornl_rsr/.

[37] G. Liu and K. Tomsovic, A full demand response model in co-optimized energy and reserve market, Electr. Power Syst. Res. 111 (2014) 62-70.

[38] The ILOG CPLEX Website, 2016. [Online]. Available: http://www01.ibm.com/software/commerce/optimization/cplex-optimizer/index.html 